\title{
SOLAR CYCLE VARIATIONS OF ELECTRON DENSITY AND TEMPERATURE IN THE VENUSIAN NIGHTSIDE IONOSPHERE
}

\author{
Robert F. Theis \\ Laboratory for Atmospheres, Goddard Space Flight Center, Greenbelt, Maryland \\ Larry H. Brace \\ Space Physics Research Laboratory, University of Michigan, Ann Arbor, Michigan
}

\begin{abstract}
The return of periapsis to low altitudes during the Summer and Fall of 1992 provided a unique opportunity for the Pioneer Venus Orbiter (PVO) to make in situ measurements in the Venusian ionosphere at much lower levels of solar activity $(F 10.7 \approx 120)$ than existed when periapsis was at low altitudes in 1979 and 1980 (F10.7 $\approx 220$ ). We present the observations of electron density $\left(\mathrm{Ne}_{\mathrm{e}}\right)$ and temperature $\left(\mathrm{T}_{\mathrm{e}}\right)$ made by the Orbiter Electron Temperature Probe (OETP) during the Entry Period. Empirical models of the $\mathrm{N}_{\mathrm{e}}$ and $\mathrm{T}_{\mathrm{e}}$ height variations are presented and compared with similar models based on OETP measurements made at solar maximum. The median $\mathrm{N}_{\mathrm{e}}$ at the ionospheric peak ( 140 $\mathrm{km}$ ) was essentially unchanged from its solar maximum value, but the ionosphere was increasingly depleted at higher altitudes, reaching a factor of 7 lower densities at $200 \mathrm{~km}$. $\mathrm{T}_{\mathrm{e}}$ was lower by almost a factor of 2 at $140 \mathrm{~km}$ but was rather significantly enhanced at higher altitudes; exceeding its solar maximum values by a factor of 1.3 at $200 \mathrm{~km}$ and a factor of 2 at $500 \mathrm{~km}$. In general these results support the earlier conclusions that the nightside upper ionosphere is depleted at lower levels of solar activity by a reduction of the nightward ion flow. The lack of $\mathrm{N}_{e}$ variation near the peak (between solar maximum and entry) suggests that nightward ion transport does not play as large a role in the peak formation as does local ion production by energetic particles. The decrease in $T_{e}$ at low altitudes suggests that the low densities of the upper ionosphere at the time of PVO entry could no longer support the conduction of heat from the dayside ionosphere, thus allowing the lower nightside ionosphere to cool by collisions with ions and neutrals, and by heat conduction to the cooler regions below.
\end{abstract}

\section{Introduction}

During the final two months or so of the Pioneer Venus Orbiter (PVO) lifetime, periapsis descended into the Venusian nightside ionosphere because of the continuing solar gravitational perturbations of its orbit. This interval, known as the Entry Period, provided a unique series of in situ measurements. The measurements were especially valuable for two reasons. First, they extended to much lower altitudes than were achieved during the low altitude operations in 1979 and 1980 when PVO last operated in the ionosphere. Second, the level of solar activity as measured at Earth was much lower $(F 10.7 \approx 120)$ than existed during the earlier period $(\approx 220)$.

Copyright 1993 by the American Geophysical Union.

Paper number 93GL03380

0094-8534/93/93GL-03380\$03.00
Since the F10.7 level was much closer to solar minimum than solar maximum, we will refer to this period as one of low solar activity. In this paper we employ data from the Orbiter Electron Temperature Probe (OETP) to investigate the effects of solar cycle variations on the nightside electron density $\left(\mathrm{N}_{e}\right)$ and temperature $\left(T_{e}\right)$. Simple empirical models are developed and compared with solar maximum models based on OETP measurements from the earlier period.

\section{The Method}

The OETP instrument and details on its operation are discussed by Krehbiel, et al. (1980), and the measurements have been used extensively to investigate the Venus ionosphere (e.g.. Brace et al. 1980, Brace et al. 1990, and Theis et al. 1984). The method employed two independent Langmuir probes (radial and axial probes). Each probe was placed at the end of a boom to measure the ambient plasma beyond the spacecraft ion sheath. The probe potentials were swept repeatedly to generate volt-ampere curves at the rate of two per second, however telemetry rate limitations returned curves to Earth at spacings of only $16 \mathrm{~s}$ or $32 \mathrm{~s}$. Typically, between 25 and 35 curves were obtained during each passage, and these were later computer-fitted to yield the measurements of $\mathrm{N}_{e}$ and $\mathrm{T}_{\mathrm{e}}$. Somewhat higher spatial resolution is available via onboard data processing, but the more accurate voltampere curve measurements are adequate for most purposes. We expect a measurement precision of the order of $5 \%$ and an absolute accuracy of $10 \%$ when $\mathrm{N}_{e}>50 \mathrm{~cm}^{-3}$. The measurements are less accurate at lower densities.

\section{Measurements and Empirical Models}

The in situ measurement coverage obtained during the final nightside sweep of PVO yielded very low altitude data that were not obtained during the 1979-80 low periapsis period. In the present study we have used the OETP data base from orbits 5011 through 5055 which provided local time coverage from $23 \mathrm{hrs}$ through $05 \mathrm{hrs}$.

Figure 1 a and $b$ show the individual $N_{e}$ and $T_{e}$ measurements from the Entry Period plotted versus altitude. The heavy solid lines represent empirical models based on medians derived from the $\mathrm{N}_{e}$ and $\mathrm{T}_{e}$ measurements. The medians were selected by first arranging the data by altitude, then identifying the median point from groups of 21 consecutive points. A given point was only selected once, even if it turned out to be the median for more than one group of 21 points. Finally, the $\mathrm{N}_{\mathrm{e}}$ and $\mathrm{T}_{\mathrm{e}}$ models were derived from the medians via standard non-linear least squares fitting 

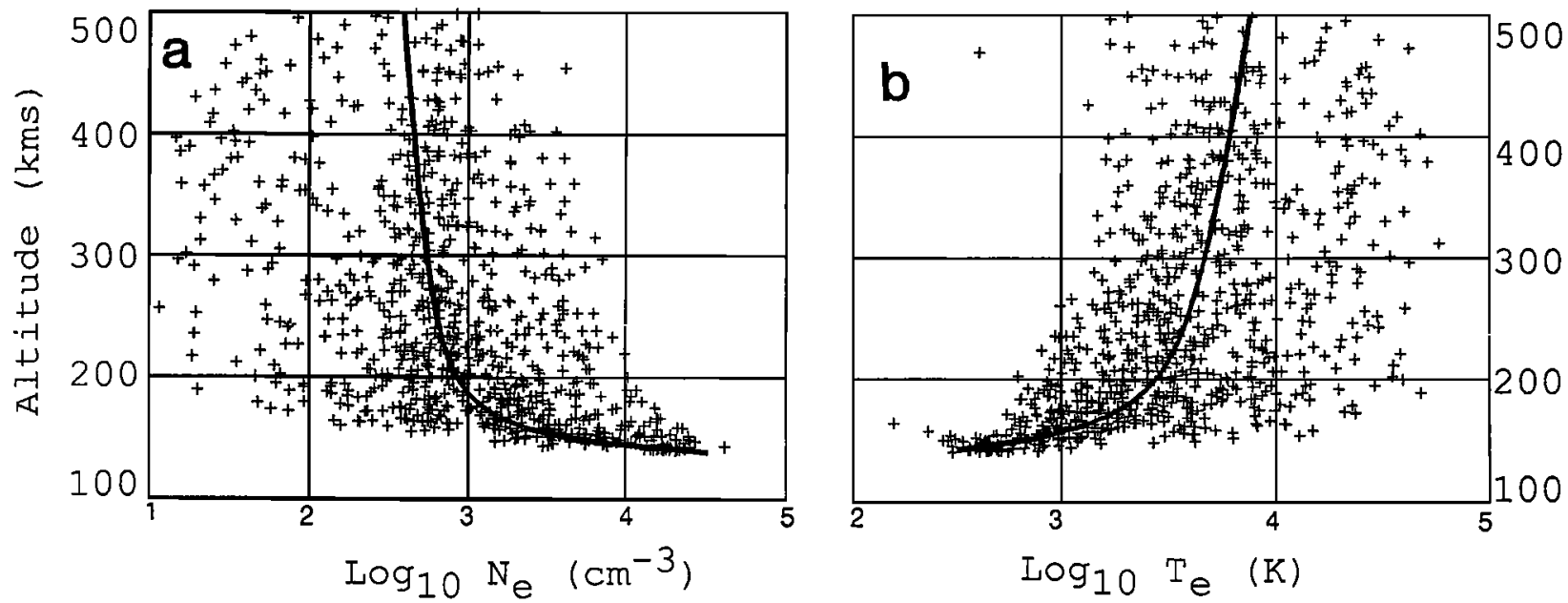

Fig.1. The $\mathrm{N}_{\mathrm{e}}$ measurements (a) and $\mathrm{T}_{\mathrm{e}}$ measurements (b) from the Entry Period. The solid lines are empirical models obtained by fitting the median values using equations 1 and 2 .

to equations of the same form as those used by Theis et al. (1984) in devising their solar maximum model of $\mathrm{T}_{e}$. The coefficients in equations 1 and 2 were the result of the fitting process.

$$
\begin{aligned}
& \log _{10}\left(N_{e}\right)=2.902+1277.7 /(h-112.6)^{2}-6.2693 \times 10^{-4} h \\
& \log _{10}\left(T_{e}\right)=3.471-1921.9 /(h-98.078)^{2}+8.5257 \times 10^{-4} h
\end{aligned}
$$

where $h$ is the altitude in $\mathrm{km}$ and $\mathrm{N}_{e}$ and $\mathrm{T}_{e}$ are in cgs units. Note that these equations cannot describe an ionospheric peak, but we would not expect to be able to model the peak region anyway because PVO did not pass through it on enough orbits of the Entry Period.

As a cautionary note, these empirical models ignore any local time variations that may be present in the 23-05 hr interval. However, local time plots of the data (not presented here) show that these are much smaller than the orbit-to-orbit and within-orbit variations, so these models may be considered representative of the median conditions at about 02 hrs.

Another important feature of the nightside ionosphere that is not captured by these models is a deep trough in $\mathrm{N}_{e}$ that was typically found between the main peak ionosphere and the upper ionosphere. Two examples of the trough are evident in the high resolution measurements shown in Figure $2 \mathrm{a}$ and $\mathbf{b}$. While this trough has not been studied carefully yet, we suspect that it has significance for the coupling between the main peak ionosphere and the upper ionosphere.

\section{Discussion}

The Entry Ionosphere. Figure 1 a evokes the following general comments about $\mathrm{N}_{e}$ at the time of entry. The region near the ionospheric peak $(140-150 \mathrm{~km})$ was much less variable from orbit-to-orbit than is the upper ionosphere. The trough between the upper and lower ionosphere (Figure 2) may be a signature of the interaction between the cold, stagnant plasma of the main peak region consisting of molecular ions and the hot, probably flowing upper ionosphere, consisting mostly of atomic ions. Measurements from other PVO instruments may later shed some light on the nature of that interaction. But before we leave this point, it should be noted that such troughs are not limited to the entry period. Brace, et al. (1980) drew attention to horizontal stratification in the nightside ionosphere, and this trough is one such feature.
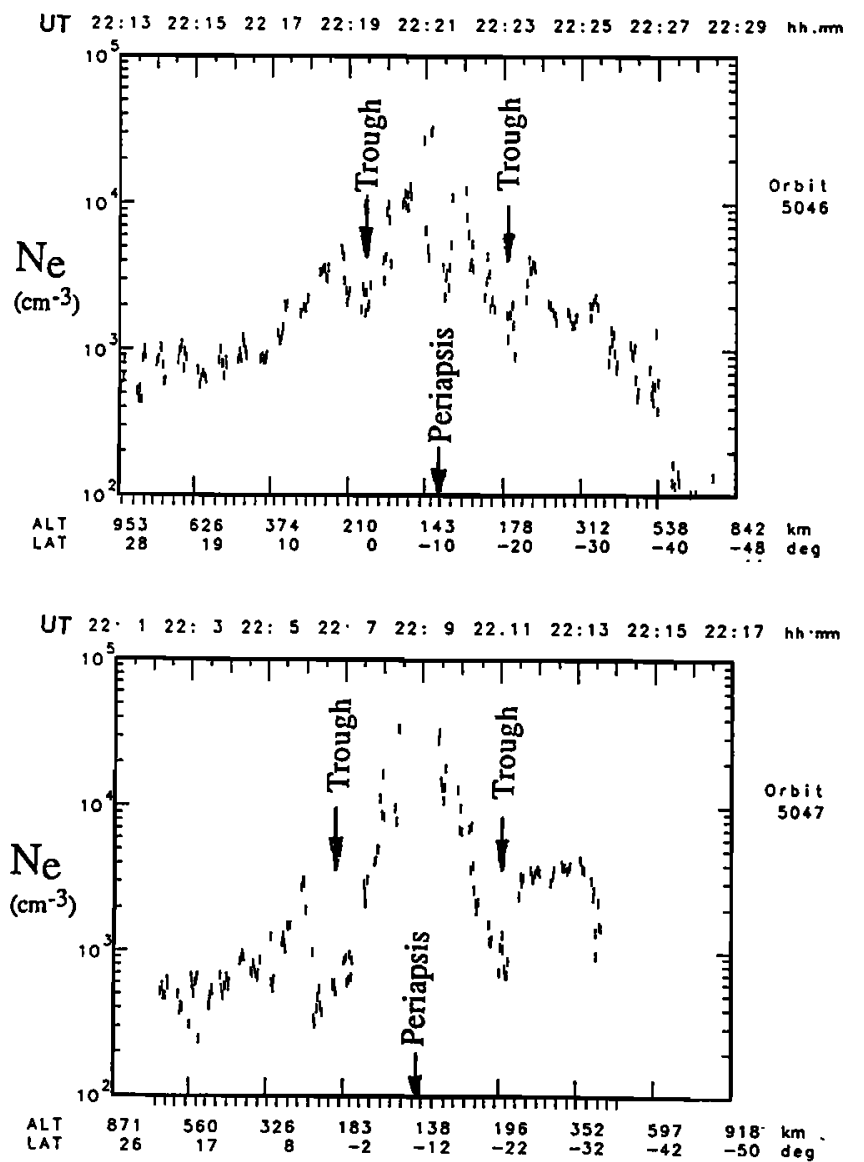

Fig. 2. High resolution $\mathrm{N}_{e}$ pass plots for orbits 5046 and 5047 showing examples of the trough between the main peak ionosphere and the upper ionosphere. The trough typically occupied the regions between about 165 and 200 kilometers, but it was highly variable from orbit to orbit. 
Another important empirical fact concerning the ionosphere at the time of entry was its great variability, particularly at altitudes above $170 \mathrm{~km}$ where the range of scatter is about a factor of 300 in $\mathrm{N}_{e}$ and a factor of 30 in $\mathrm{T}_{e}$. Although the relationship between $\mathrm{N}_{e}$ and $\mathrm{T}_{e}$ is not evident in Figure 1, it becomes quite clear when examining individual orbit profiles that the scatter arises both from spatial structure and from orbit-to-orbit variations. It is also clear from individual pass plots that $\mathrm{N}_{e}$ and $\mathrm{T}_{e}$ were strongly anticorrelated.

Solar Cycle Variations. While a quantitative evaluation of the ionosphere at the time of PVO entry cannot be done until the data from other instruments are available, perhaps the OETP results alone can provide a useful start on the question of solar cycle variations and their causes. Since PVO did not survive through solar minimum, we will not be able to define the full range of ionospheric response, but the average solar activity at entry (F10.7 $\approx 120)$ is much closer to a typical minimum $(\approx 70)$ than to the maximum of $1980(\approx 220)$. Therefore comparisons between the entry measurements and the 1979-80 measurements should capture the major trends.

To facilitate this comparison, Figure $3 \mathrm{a}$ and $\mathrm{b}$ show the entry model profiles of $\mathrm{N}_{e}$ and $\mathrm{T}_{\mathrm{e}}$ (solid lines in Figure $1 \mathrm{a}$ and b) and the solar maximum model profiles (Theis et al., 1984) for the same solar zenith angles ( $\mathrm{SZA}=143^{\circ}$ or $\mathrm{LT}=2.5 \mathrm{hrs}$ ). A cursory comparison of the entry data with solar maximum data yields the following generalizations about their differences. The upper ionosphere $(\mathrm{h}>170 \mathrm{~km})$ was heated and depleted at the lower levels of solar activity existing at entry. $\mathrm{N}_{\mathrm{e}}$ near the peak $(\mathrm{h} \approx 140-150 \mathrm{~km}$ ) responded very little to the change in solar activity, but $T_{e}$ in the peak region was much lower.

The apparent lack of $\mathrm{N}_{\mathrm{e}}$ solar cycle variation at the peak was also found by Kar et al.(1993) who used entry period ion mass spectrometer (OIMS) measurements. However, both the OETP and OIMS measurements near the peak may be in conflict with the findings of Kliore et al. (1991) who used PVO radio occultation measurements to study the solar cycle variation between 1979 and 1986. They found the peak $\mathrm{N}_{e}$ was higher by about a factor of 1.5 at solar maximum relative to the intermediate levels of solar activity in 1983, and a factor of 2 higher than at solar minimum in 1986. Since these data refer to a different solar cycle, it is not clear whether or not the discrepancy is a real one. In either case, however, there was not very much solar cycle variation in $\mathrm{N}_{e}$ at the peak.

Solar Cycle Variations in $\mathrm{N}_{\mathrm{e} .-}$ The fact that $\mathrm{N}_{\mathrm{e}}$ in the vicinity of the main peak did not change much between solar maximum and entry has implications for the relative roles of nightward ion transport and local energetic electron precipitation in its formation. Knudsen et al. (1987), with the help of PVO radio occultation measurements, concluded that the reduction in the dayside $\mathrm{Ne}_{\mathrm{e}}$ at solar minimum could be expected to cause the ionopause there to move planetward, thus cutting off the nightward flow of ions. Since nightward flow of $\mathrm{O}^{+}$is believed to be a major source for maintaining the nightside ionosphere (Spenner et al., 1981, Kliore et al., 1991).

Returning to the entry measurements at the peak, it is somewhat surprising that $\mathrm{N}_{\mathrm{e}}$ differed little from its solar maximum value. One would have expected the reduced nightward flow to have produced a detectable reduction in the peak density. Indeed, the radio occultation measurements of Kliore et al. (1991) from the previous solar cycle confirmed this. The absence of such a reduction at entry suggests that nightward flow may not have been as important in the formation of the peak at that time. An alternative explanation might be that local ion production by energetic electron precipitation (Gringauz et al., 1979) actually was higher at the time of PVO entry by just enough to counter the effects of the reduced nightward transport. Clearly, the solar cycle variation of the peak region (or the lack of it) is a matter for more careful future investigation in which the measurements from several PVO instruments are combined.

Unlike the peak $N_{e}$ behavior, the density of the upper nightside ionosphere shows a huge solar cycle response. Comparison of the models in Figure 3 shows that the median $\mathrm{N}_{e}$ at entry was lower by a factor of between 5 and 8 at altitudes above $200 \mathrm{~km}$. This depletion was also consistent with observed solar cycle variations of the upper nightside ionosphere or the ionotail (e.g. Knudsen et al., 1987, Brace et al., 1990).
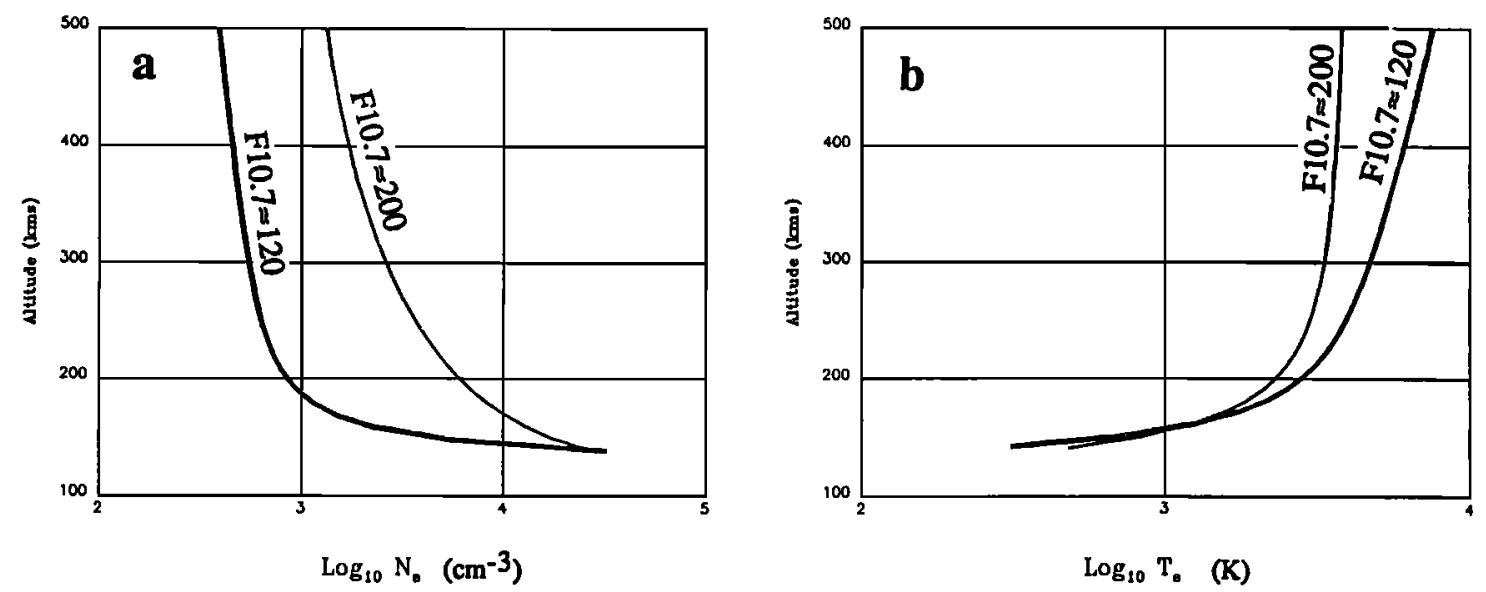

Fig. 3. Comparison of the Entry Period models $(\mathrm{F} 10.7 \approx 120)$ and solar maximum models (F10.7 $\approx 200$ ). $N_{e}$ at the peak changed little, while $T_{e}$ was much lower. At altitudes above $170 \mathrm{~km}$, $\mathrm{N}_{\mathrm{e}}$ was lower and $\mathrm{T}_{\mathrm{e}}$ was higher at Entry. These changes can be understood to first order by invoking (at low solar activity) a partial cutoff of nightward ion flow and heat conduction from the dayside ionosphere. 
Additional differences between solar maximum and entry are that the ionopause at entry occurred at much lower altitudes and "disappearing ionospheres" were more common (Cravens et al., 1982). A preliminary examination of the solar wind measurements (personal communication, Mihalov, 1993) suggests that variations in solar wind dynamic pressure are a factor in this variability.

Solar Cycle Variations in $\mathrm{T}_{\mathrm{e}}$. The temperature of the nightside ionosphere was quite different at entry; $\mathrm{T}_{e}$ being lower below $170 \mathrm{~km}$ and enhanced above that relative to solar maximum. The median $\mathrm{T}_{\mathrm{e}}$ at $140 \mathrm{~km}$ (near the peak) was a factor of 2 colder than at solar maximum, but exceeded its solar maximum value by a factor of 1.3 at $200 \mathrm{~km}$ and a factor of 2 at $500 \mathrm{~km}$. However, the examination of individual orbits shows that $T_{e}$ was considerably higher on many passages in which $\mathrm{N}_{\mathrm{e}}$ was heavily depleted. These higher temperatures are reminiscent of the "disappearing ionospheres", although the heat source has not been identified. Perhaps the explanation lies in the fact that the depleted upper ionosphere is thermally connected to the hot plasma of the mantle or the magnetosheath, rather than to the cool underlying ionosphere. This explanation is consistent with the tendency to find largely horizontal magnetic fields in the disappearing ionospheres, but this effect has not been verified for the depleted ionosphere orbits of the entry period.

The lower median $T_{e}$ found in the peak region is a puzzle, as it implies a reduction in the nightside heat source at lower levels of solar activity even though there appears to be enough local ion production to maintain a large nightside main peak. Apparently the heat source was not primarily local precipitation and the resulting secondary electrons. Hoegy et al. (1980), using solar maximum data, invoked electron heat conduction from the dayside ionosphere to explain the elevated $T_{e}$ on the nightside. The observed reduction in $\mathrm{T}_{\mathrm{e}}$ at entry suggests that the conduction path into the hot dayside ionosphere may have been missing, or less effective at these lower levels of solar activity. This would be consistent with the expectation that the decline in altitude of the terminator ionopause at low solar activity would reduce not only the nightward ion flow but inhibit heat conduction from the dayside ionosphere. Conduction becomes less efficient at the low densities that are left behind when the ionopause moves inward at solar minimum. Cutoff from its dayside heat source, the lower ionosphere cools locally to the ions and thermospheric neutrals, and by conduction into the even colder underlying atmosphere.

These ideas may be difficult to fully check out, since PVO's fuel did not last long enough to permit low altitude measurements of the dayside ionosphere. However, there were a few measurements a month or so earlier at altitudes near $200 \mathrm{~km}$ as periapsis swept through the terminator region. These data are expected to shed light on the ionopause and upper ionosphere behavior in this region at similarly low levels of solar activity. This should permit some of the above preliminary interpretations to be evaluated further.

\section{References}

Brace, L. H., R. F. Theis, W. R. Hoegy, J. H. Wolfe, C. T. Russell, R. C. Elphic, A. F. Nagy, The Dynamic Behavior of the Venus Ionosphere, J. Geophys. Res. 85. 7663, 1980.

Brace, L. H., R. F. Theis, and J. D. Mihalov, The Response of the Venus Nightside Ionosphere and Ionotail to Solar EUV and Solar Wind Dynamic Pressure, $\mathrm{J}$. Geophys. Res., 95, 4075, 1990.

Cravens, T. E., L. H. Brace, H. A. Taylor, C. T. Russell, W. C. Knudsen, K. L. Miller, A. Barnes, J. D. Mihalov, F. L. Scarf, S. J. Quenon and A. F. Nagy, Disappearing Ionospheres on the Nightside of Venus, ICARUS. 51. $271,1982$.

Gringauz, K. I., M. I. Verigin, T. K. Breus, and T. Gombosi, The Interaction of Electrons in the Optical Umbra of Venus with the Planetary Atmosphere - The Origin of the Nighttime Ionosphere, J.Geophys. Res. $\underline{84}, 2123,1979$.

Hoegy, W. R., L. H. Brace, R. F. Theis, and H. G. Mayr, Electron Temperature and Heat Flow in the Nightside Venusian Ionosphere, J. Geophys, Res.. 85. 7811, 1980.

Kar, J., R. E. Hartle, and J. M. Grebowsky, Evidence of the Dominance of Electron Impact Ionization in Producing the Nightside Ionospheric Peak from PVO/OIMS Measurements at Solar Minimum, EOS, 74, No. 16, 187, 1993.

Kliore, A. J., J. G. Luhmann, Martina H. G. Zhang, The Effect of the Solar Cycle on the Maintenance of the Nightside Ionosphere of Venus, J. Geophys. Res. 96. No. A7, 11065, 1991.

Knudsen, W. C, A. J. Kliore and R. C. Whitten, Solar Cycle Changes in the Ionization Sources of the Nightside Venus Ionosphere, J. Geophys. Res. 92. 13391, 1987.

Krehbiel, J. P., L. H. Brace, J. R. Cutler, W. H. Pinkus, R. B. Kaplan, Pioneer Venus Orbiter Electron Temperature Probe, IEEE Transactions on Geoscience and Remote Sensing, GE-18, 49, 1980.

Spenner, K., W. C. Knudsen, R. C. Whitten, P. F. Michelson, K. I. Miller, and V. Novak, On the Maintenance of the Venus Nighttime Ionosphere: Electron Precipitation and Plasma Transport, $\mathbf{J}$. Geophys. Res. 86. 9170, 1981.

Theis, R. F., L. H. Brace, R. C. Elphic and H. G. Mayr, New Empirical Models of the Electron Temperature of the Venus Ionosphere with application to Transterminator Flow, J. Geophys. Res.. 89, 1477, 1984.

L. H. Brace, Space Physics Research Laboratory, University of Michigan, Ann Arbor, MI 48109

R. F. Theis, Laboratory for Atmospheres, NASA/Goddard Space Flight Center, Code 910, Greenbelt, MD 20771

(Received: June xx,1993; revised August 6, 1993; accepted 1993.) 